Western University Scholarship@Western

Electrical and Computer Engineering Publications Electrical and Computer Engineering Department

$8-2010$

\title{
Integrating Recommender Information in Social Ecosystems Decisions
}

Renato A C Capuruco

Western University, r.capu@uwo.ca

Luiz Fernando Capretz

University of Western Ontario, lcapretz@uwo.ca

Follow this and additional works at: https://ir.lib.uwo.ca/electricalpub

Part of the Computer Engineering Commons, Electrical and Computer Engineering Commons, and the Systems Architecture Commons

Citation of this paper:

Capuruco R.A.C. and Capretz L.F. Integrating Recommender Information in Social Ecosystems Decisions, 4th European Conference on Software Architecture, Copenhagen, Denmark, pp. 143-150, DOI: 10.1145/1842752.1842783, ACM Press, August 2010. 


\section{Integrating Recommender Information in Social Ecosystems Decisions}

\author{
Renato A C Capuruço \\ University of Western Ontario, Dept of Electrical and \\ Computer Engineering \\ London, Ontario, CANADA, N6A 5B9 \\ r.capu@uwo.ca
}

\author{
Luiz F Capretz \\ University of Western Ontario, Dept of Electrical and \\ Computer Engineering \\ London, Ontario, CANADA, N6A 5B9 \\ Icapretz@eng.uwo.ca
}

\begin{abstract}
The exploration of online social networks whose members share mutual recommendations and interactions is a time-dependent and contextual-based process which aims to predict the social status among members, ultimately improving the network's discoverability to achieve societal gain. To address the difficulties associated with the process, this article presents an integrated recommender model whose statements are time-dependent, interaction-aware, and social context-sensitive. The originality of the proposed model stems from the integration of the predictive recommender, social networks, and interaction components. Each model is developed based on: (1) a time history and decay algorithm to consider the decreasing intensity of recommendations among members over time; (2) a predictive aggregating function for improved assessment of recommendations for various social contexts; and, (3) a homophily algorithm to estimate the degree in which a recommender-based contact between similar people occurs to dissimilar people. Details of the framework are described, a recommender search strategy methodology is devised, and a case study is used to demonstrate its capabilities. Possible extensions are then outlined.
\end{abstract}

\section{Keywords}

Recommender System, Social Network, Homophily, Time Decay

\section{INTRODUCTION}

The basic consideration in an effective online community is the opportunity that the social presence may provide to achieve societal gain, in addition to satisfying various relations constraints and membership requirements. In general, online sociality can be viewed as a complex proliferation of social computing technologies and resources that has many social applications ranging from e-commerce and entertainment to social libraries and networking sites. Despite the many facets of social systems, there has been a tendency to build those around certain particular models. Implementations based on the fundamentals of Recommender Systems [15] are widely used online to suggest to users those items they may like or find useful based on recommendations from other people. The information that other people provide may come from explicit ratings, tags, reviews, or implicitly from how they conduct themselves in the online environment. Collaborative Filtering [7] is the most commonly used technique, which provides the means for information aggregation, selection, filtering, and sorting. Social network approaches, on the other hand, provide a form of social computing that is intrinsically more interaction-based rather than discoveryoriented. A recent example is a model for mobile social networks
[12] as a tool that supports interaction among networked mobile users. It uses graph theory [17] and psychological concepts to design mobile social services. In recent years, there has been increased research interest to incorporate sorts of social presence into recommendation environments. As examples, a trust propagation model was used in [13] to improve recommendations of generic items, and a recommender architecture that is contextaware was proposed in [1] to improve travel planning; such efforts complicate and render traditional methodologies as insufficient to deal with the distinct formulation involved.

In most social computing-related models, such as those described earlier, the social interactions and recommendations are often represented separately, lacking time-dependent social contexts characterization, and dependences among these are overlooked. Also, social networks that are formed from those models provide few guidelines regarding their standing when related information and patterns are expressed over time and articulated based on criteria of community membership. Furthermore, decisions related to the desired importance of interrelationships among members are left unattended, and the models do not incorporate mechanisms to address the rational of such decisions. These issues represent modeling difficulties and limitations that need to be addressed in a realistic social computing model in order to provide superior exploration and discovery of social spaces.

In an attempt to address the preceding challenges, this paper presents an integrated model for online communities' discovery and exploration. The developments made in this article build upon on a newly developed model by Capuruço and Capretz [4]. The model requires inputs of all members and their relationships and included a multi-disciplinary algorithm for relations assessment. To add practicality and supplement the user's subjective judgment, three main components have been incorporated into the model to form the enhanced framework presented in this article: (1) a time history and decay algorithm to consider temporal recommendations and interactions; (2) a predictive-aggregating function for different types of social contexts; and, (3) a homophily algorithm to evaluate people's interconnections proximity. Details of these components are described along with their combined implementation. A prototype application is then demonstrated and its performance validated.

\section{PROPOSED FRAMEWORK}

The main components of a social computing recommender model (SCRM) that incorporates a time-dependent, interactionaware, and social context-sensitive modules are (Figure 1):

- Detailed SCRM models (computer-mediated interaction, social network relationships, and recommendation-based perceptions of taste or opinions) 


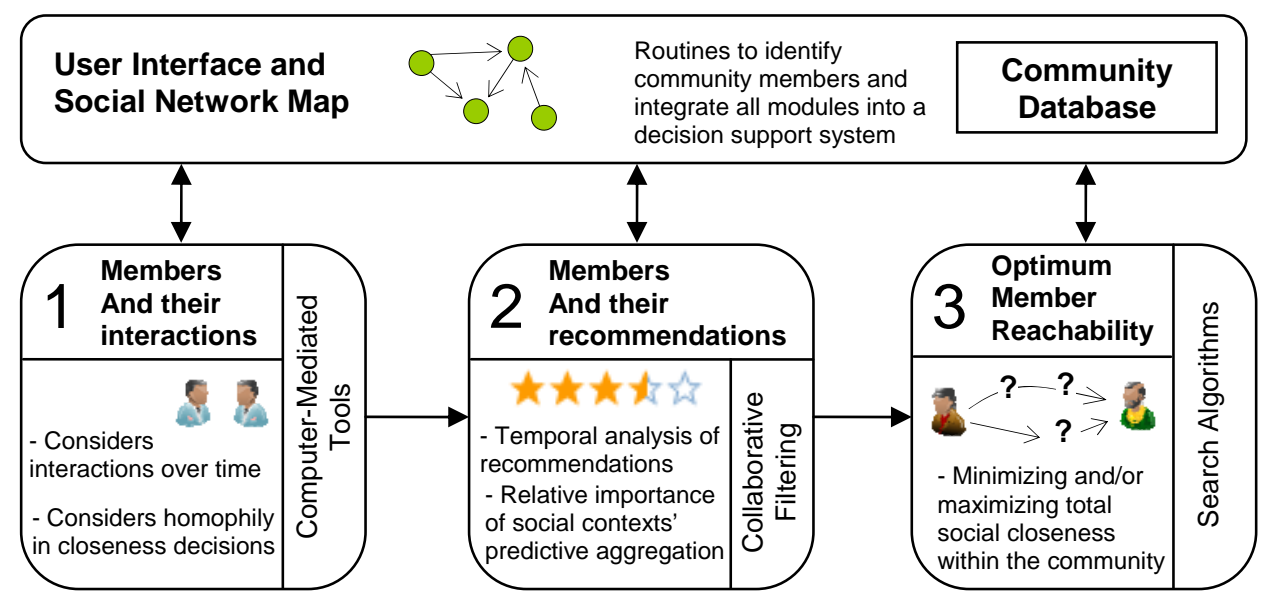

Figure 1. Components of the proposed model.

- SCRM Constraints (social context, social relation, social recommendation, user defined constraints, such as time horizon, decay intensity, etc.)

- SCRM Decision Support Module (user interface, community database, social closeness assessment, recommender search, visualization)

\subsection{Model Components}

At the core of a successful SCRM are proper models for eliciting the interactions and capturing the relationship patterns of the individuals in the community. This allows the several recommendation statements to be evaluated according to the network that is formed, which helps derive recommender-aware social status indicators. As a result, the benefits of knowing those patterns and conditions can be returned to the community itself by the means of a search engine that optimally reaches a member from any interrelated member.

\subsubsection{Recommender Model}

This model is composed by uniquely identifiable members that express opinions of taste and rating values on other uniquely identifiable members. All the information may or not be made public to every other member and every member is able to express them whenever they prefer. Members express recommendations values in members based on their perceived quality as source of advise about past and current topics of discussion. For example, a member should recommend another member if she/he likes one's opinions, behavior, contribution, etc. to the overall development of the community.

Every member can express one or more recommendation statements that embed his/her opinions about the likability of another member anytime in any social dimension. Recommendations statements have the following form:

- Recommendation(FromMember,ToMember).value

- Recommendation(FromMember,ToMember).date

- Recommendation(FromMember,ToMember).context

Every member's recommendation statement can be formalized in a recommender function whose domain is $M$ and whose co- domain is $[1,5]$ where 1 means total dislike and 5 total like. A missing value (i.e., function not defined) represents the fact that the member has not expressed a recommendation statement about another member. This is as follows:

$$
r_{\left[\text {member }_{\mathrm{m}} \text {, } \text {,ate }_{\mathrm{d}} \text {, context }_{\mathrm{c}}\right]}: \mathrm{M} \rightarrow[1,5] \cup \perp
$$

For example, $r_{[25,39448,3]}\left(m_{3}\right)=4.55$ means that member $m_{25}$ issued a recommender statement on 01/Jan/2008 (39,447 days after January 1,1900$)$ and rated member $m_{3}$ as 4.55 in the social context 3 (e.g., workplace), a very high recommendation rating expressing his/her almost complete likeability for the member.

\subsubsection{Interaction Model}

This model is composed by uniquely identifiable members that express contact with other uniquely identifiable members. All the information may or not be made public to every other member and every member is able to make contact with any other member whenever they prefer. Members express mutual relationships with members based on their actual communication patterns within past and current topics of discussion. For instance, a member should contact (e.g., though e-mailing, discussion board posting, blogging, etc.) another member if she/he interconnects with one's opinions, behavior, contribution, etc.

Every member can express one or more interaction statements that embed his/her contacts with another member anytime in any social dimension. Recommendations statements have the following form:

- Interaction(FromMember,ToMember).value

- Interaction(FromMember,ToMember).date

Every member's interaction statement can be formalized in an interaction function whose domain is also $M$ and whose codomain is $[x]$ where $x$ is a calculated value that measures the social distance (i.e., Closeness) between two members and it is a function of the Social Status that each member shares in the network. This is explained in more detail in the next subsections. A missing value (i.e., function not defined) represents the fact that the member has not expressed an interaction statement with another member (e.g., "members have not met"). This is as follows: 


$$
i_{\text {member } \left._{\mathrm{m}}, \text { date }_{\mathrm{d}}\right]}: \mathrm{M} \rightarrow[\mathrm{x}] \mathrm{U} \perp
$$

For example, $i_{[33,39062]}\left(m_{6}\right)=2.78$ means that member $m_{33}$ issued an interaction statement on 12/Dec/2006 (39,062 days after January $1,1900)$ and the calculated social distance to member $m_{6}$ is 2.78 . The order of magnitude of the social distance's calculated value is relative to the recommender function's co-domain boundaries because Closeness is calculated from the recommendations; therefore, inheriting its scale.

Interactions are considered to be the main avenue upon which recommendations are passed over from one member to another. The matter of fact is that, in this model, it takes one and only one formal interaction between a pair of members to broadcast all (un)known statements of recommendations given over time and in different social contexts by the community. These two aspects are very important features to consider because it not only allows capturing local recommendations (e.g., from members and their immediate neighbors), but also global opinions (e.g., from anyone to everyone else); therefore, bringing all the community together to validate all of the recommendations.

\subsubsection{Social Network Model}

A social networks perspective is based on the theoretical constructs of sociology and mathematical foundations of graph theory. Classic research in "sociograms" and "sociometric" [14] established the typical analyses and mathematical models [9] that are used today to understand and analyze social network data.

Based on these generalizations about the features of personal networks combined with the previously introduced formulations for interactions and recommendations, the basic concepts upon which this model is constructed has emerged as follows:

- A graph $G$ consists of a finite nonempty set $V=V(G)$ of $p$ nodes together with a prescribed set $X$ of $q$ unordered pairs of distinct nodes of $V$. Each pair $x=\{u, v\}$ of nodes in $X$ is an edge $e$ of $G$ and $x$ is said to join $u$ and $v$;

The proposed social network $N$ is a graph where each node $p$ is a member $m$. Each pair $x=\left\{m_{\mathrm{u}}, m_{\mathrm{v}}\right\}$ of members in $N$ is an edge $e$ of $N$ and $x$ is said to join $m_{\mathrm{u}}$ and $m_{\mathrm{v}}$; a numerical value, $f(e)$, is assigned to each edge $e$, which is a measure of Social Closeness. The complete environment is composed by (Figure 2):

- A set $M$ of $m$ uniquely identifiable members:

$$
\mathrm{M}=\left\{\mathrm{m}_{1}, \mathrm{~m}_{2}, \mathrm{~m}_{3} \ldots \mathrm{m}_{\mathrm{m}-1}, \mathrm{~m}_{\mathrm{m}}\right\}
$$

- A set $R$ of $n$ uniquely identifiable recommendation statements:

$$
\mathrm{R}=\left\{\mathrm{r}_{1}, \mathrm{r}_{2}, \mathrm{r}_{3} \ldots \mathrm{r}_{\mathrm{n}-1}, \mathrm{r}_{\mathrm{n}}\right\}
$$

- A set $I$ of $k$ uniquely identifiable interaction statements:

$$
\mathrm{I}=\left\{\mathrm{i}_{1}, \mathrm{i}_{2}, \mathrm{i}_{3} \ldots \mathrm{i}_{\mathrm{k}-1}, \mathrm{i}_{\mathrm{k}}\right\}
$$

- A set $C$ of $j$ characteristics associated with each member $m$ of $M$ :

$$
\mathrm{C}=\left\{\mathrm{c}_{1}, \mathrm{c}_{2}, \mathrm{c}_{3} \ldots \mathrm{c}_{\mathrm{j}-1}, \mathrm{c}_{\mathrm{j}}\right\}
$$

- A Social Status function $s(m)$ associated with each member $m$ of $M$ :

$$
\mathrm{S}(\mathrm{m})=\mathrm{f}\left(\mathrm{R}_{\text {predicted }}\right)_{\mathrm{m}}
$$

- A Social Distance function $d(i)$ associated with each interaction $i$ of $I$ from members $m_{a}$ to $m_{b}$ of $M$ :

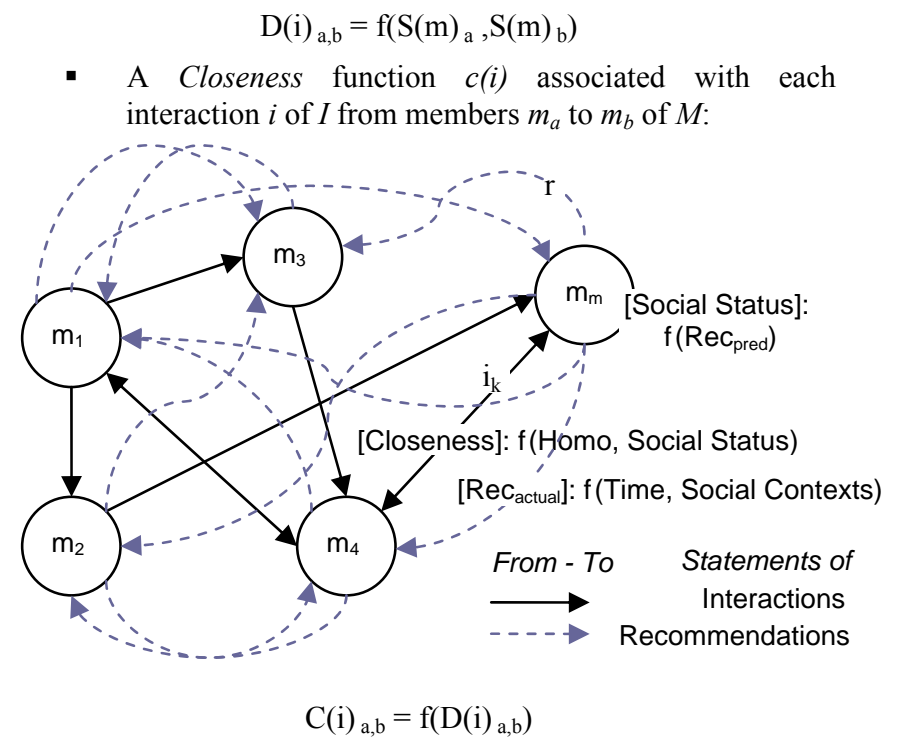

Figure 2. Web of interactions and recommendations representation

\subsubsection{Closeness Assessment Model}

The assessment model aims at supporting three difficult decisions related to social network exploration, each lending itself well to a different solution mechanism.

First, the process of considering network activities that are dynamic and time-dependent in nature. As such, a time history and decay scheme can aid in the process of harmonizing them to the present time.

Second, unknown localized (between any given pair of members) and a global (among all members) recommendation statements is an issue that may worsen the calculation of a particular's member social status, and as such, improving the accuracy of those calculations is a problem that involves prediction and lends itself well to collaborative filtering application.

Third, the people's social network and implications to the information they receive, the attitude they form, and the interactions they experience sets the stage and contexts for the formation of social spaces in which homophilous relations form and flourish. This is a difficult problem that lends itself well to the consideration of a wide range of socio-demographic and behavioral dimensions to account for the impact of multiplex ties on the dynamics of the network change over time.

Integrating these three components together derives a 5-step calculation. These five calculation steps form the Closeness assessment model with each component / sub-model dealing with one of the sub-problems using a different technique.

\subsubsection{Time History and Decay}

The main goal of the time history and decay sub-model is to account for recommendations expressed in different points in time. It calculates a decay factor which accounts for the decreasing effect of importance of a statement of recommendation in different time spans. The proposed model is set up according to the intuition that older statements of recommendation worth less than newer expressed ones. 
More precisely, according to the general definitions given by Cohen and Strauss [6] for decay functions, consider a stream of recommendations where $f(t) \geq 0$ is the recommendation value of the stream obtained at time $t$. For sake of simplicity, it is assumed that the stream only receives values at discrete times, and therefore, $t$ is integral. A decay function $g(x) \geq 0$ defined for $x \geq 0$ is a non-increasing function. At time $T$, the weight of the item that arrived at time $t \leq T$ is $g(T-t)$ and the decayed value is $f(t) g(T-t)$. From that, it is obtained the decayed sum of $f(t)$ under the decay function $g(x)$ that is defined as follows:

$$
V_{g}(T)=\sum f(t) g(T-t), \perp t \leq T
$$

A generalized representation of the above formulation is as follows (Figure 3):

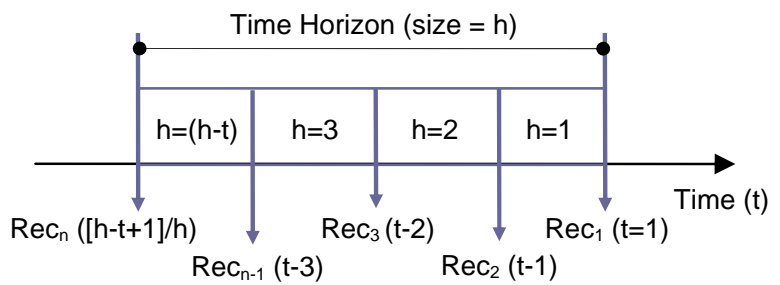

Figure 3. Time history and decay algorithm representation

Assuming $n$ recommendations are to be considered up to a maximum time horizon $h$, a recommendation at time $t$ from the most recent recommendation will have a decayed recommender factor of $(h-t+1) / h$. Recommendations that are not reachable within the maximum time horizon have no decayed value.

As an example, let's suppose a member $m$ has had 9 recommendations in the course of 5 years of being in a community. Considering a time horizon $\mathrm{t}=4$ years, let's say that only 4 of those 9 statements fall within this horizon, one per year: in this case, each of the 4 affected recommender metrics would be multiplied by factors of $(4-1+1) / 4=1$ for the most recent, (4$2+1) / 4=0.75$ for the $2^{\text {nd }}$ most recent, $(4-3+1) / 4=0.50$ for the $3^{\text {rd }}$ most recent, and $(4-4+1) / 4=0.25$ for the oldest one, respectively.

In this way, a linear decay propagation function is adopted: newest member's recommendations have proportionally higher importance than older ones in accordance with the number of recommendations in the time horizon.

\subsubsection{Predictive Aggregation}

The predictive aggregation sub-model is made necessary for two main reasons: (1) to reduce the dimensionality of the many recommendations to a singular aggregated value that can be used for further processing, and (2) to enhance the calculation of the Social Status function by predicting missing local and global recommendations.

First, the aggregation calculation engine takes as input the recommendation matrix (representing all the community recommender statements) and produces, as output, an equal matrix of pondered recommendations. This is preformed by using either of two approaches: member- and context-centric (Figure 4).

The member-centric approach takes as input the recommender network as a $M \times M$ matrix where the recommender value $r$ on each cell $i, j$ (if present) represents the recommender rating from member $m_{i}$ to $m_{j}$. Because every recommender statement in the matrix refers to a certain context $c$ only, $n$ input matrices are generated, one for each context. Next, each context is assigned a degree of importance $w$ (weight) and the pondered rating $r$ ' of member $i$ to member $j$ is the weighted sum of the ratings in each context $c$. More precisely:

$$
r_{i, j}^{\prime}=\sum_{i=1}^{m} \sum_{j=1}^{m}\left(\frac{\sum_{c=1}^{n}\left(r_{i, j} \times w_{c}\right)_{c}}{\sum_{c=1}^{n} w_{c}}\right)
$$

As such, a single output matrix $M \times M$ is produced.

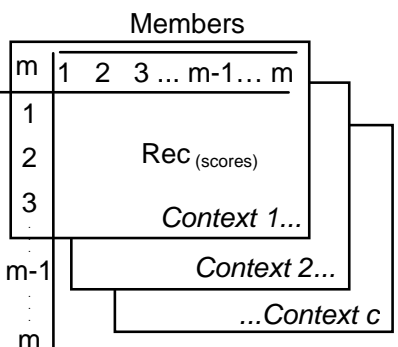

(a)
Social Contexts

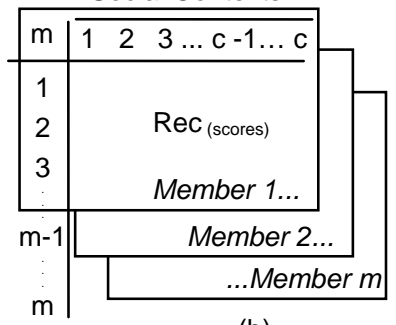

(b)
Recommendations that are unknown for all social contexts remain unidentified and are excellent candidates for prediction.

Figure 4. (a)Member- and (b) Context-centric prediction approaches

The context-centric approach takes as input the recommender network as a $M \times C$ matrix where the recommender value $r$ on each cell $i, j$ (if present) represents the recommender rating received by member $m_{i}$ in the context $\mathrm{c}_{j}$. Because every recommender statement in the matrix refers from a certain member $m$ only, $n$ input matrices are generated, one for each member. Next, the pondered rating $r$ ' of member $i$ in context $j$ is the simple average of the ratings received from each member $m$. More precisely:

$$
r_{i, j}^{\prime}=\sum_{i=1}^{m} \sum_{j=1}^{c}\left(\frac{\sum_{m=1}^{n}\left(r_{i, j}\right)_{m}}{n}\right)
$$

As a result, a single output matrix $M \times C$ is produced. Recommendations that were not received from all members remain unknown and are excellent candidates for prediction.

Either of the previous outcome matrices is the traditional input to a Collaborative Filtering $(\mathrm{CF})$ algorithm whose main function is to predict the missing recommendation values. If the membercentric approach matrix is to be used, it lends itself very well to compute missing recommendations against every other member. On the other hand, the context-centric approach matrix is mainly geared towards the computation of missing recommendations taking into consideration dependences among different contexts. This is achieved by using CF's classical steps, as follows:

- Similarity Metric: The goal is to calculate the correlation of two overlapping members (represented as vectors of ratings), outputting a $m \times m$ Member Similarity matrix in which $i$ th row contains the similarity values of $i$ th member against every other 
member. Pearson's correlation coefficient (7) is the most used technique, as follows:

$$
p_{a, u}=\frac{\sum_{i=1}^{m}\left(r_{a, i}-\bar{r}_{a}\right)\left(r_{u, i}-\bar{r}_{u}\right)}{\sqrt{\sum_{i=1}^{m}\left(r_{a, i}-\bar{r}_{a}\right)^{2} \sum_{i=1}^{m}\left(r_{u, i}-\bar{r}_{u}\right)^{2}}}
$$

Both positive and negative similarities values are calculated because similar and dissimilar members $u$ to the current member $a$ are important measures to grasp the overall community feeling about $a$ and, therefore, cannot be ignored.

- Rating Predictor: The predicted recommendation rate of member $i$ for the current member $a$ is the weighted sum of the ratings given to member $i$ by the $k$ neighbors of $a$. This is the classical CF's last step, as follows:

$$
r_{a, i}=\bar{r}_{a}+\frac{\sum_{u=1}^{k} p_{a, u}\left(r_{u, i}-\bar{r}_{u}\right)}{\sum_{u=1}^{k} p_{a, u}}
$$

\subsubsection{Social Status}

Once all missing recommendations have been predicted, it is necessary to compute the Social Status for each member. If the member-centric approach was used, hence producing a matrix of size $M \times M$, the Social Status $s_{a}$ for each member $m_{a}$ is the simple average of the ratings given to this member by all of the other members (except itself), as follows:

$$
s\left(m_{a}\right)=\frac{\sum_{i=1}^{m} r_{a, i}}{m-1} \perp a \neq i
$$

Alternatively, if the context-centric approach was used, hence producing a matrix of size $M \times C$, each context $c$ in $n$ number of social contexts is assigned a degree of importance $w$ (weight). Next, the Social Status $s_{a}$ for each member $m_{a}$ is the weighted sum of the ratings in each context $c$, as follows:

$$
s\left(m_{a}\right)=\frac{\sum_{c=1}^{n}\left(r_{a, c} \times w_{c}\right)}{\sum_{c=1}^{n} w_{c}}
$$

\subsubsection{Social Distance}

The social distance $d$ represents the perceived strength of the relationship between a pair of members. It is a direct function of Social Status, and as such, its computation simply averages out the predicted Social Statuses $s_{i}$ and $s_{j}$ of a pair of interconnected members $m_{i}$ and $m_{j}$, respectively. This is shown as follows:

$$
d_{i, j}=\frac{s\left(m_{i}\right)+s\left(m_{j}\right)}{2}
$$

\subsubsection{Homophily Computation}

The main goal of the homophily sub-model is to adjust the social distance "Closeness" between a pair of members based on their reciprocal interaction and similarity of personality attributes. Defined by Lazarsfeld and Merton [2], the homophily theory states that most human communication will occur between a source and a receiver who are alike. Homophily implies that distance in terms of social characteristics translates into network distance, the strength of relationships (i.e., interactions) through which a piece of information (i.e. recommendation) must travel to connect two individuals.

More specifically, the homophily computation takes as input the calculated Social Distance $d$ from member $m_{i}$ to $m_{j}$ and assigns a degree of importance $w$ (weight) to each of their matching homophily feature $h$ of $n$ available features. Next, an overall homophily coefficient $c$ is calculated as a product of each $w_{h}$ to represent the extent by which each original social distance $s$ from member $i$ to $j$ should be shortened. Then, the adjusted distance $d$ from member $i$ to member $j$ is $d \times c$. More precisely:

$$
d_{i, j}^{\prime}=\left(\prod_{h=1}^{n} w_{h}\right) \times d_{i, j} \perp h_{i}=h_{j}
$$

As an example, initial network studies showed substantial homophily by demographic characteristics like age, gender, race/ethnicity and education ([2], [11]) and by psychological characteristics like intelligence, attitudes, and aspirations [16]. Considering that two members in a community have a calculated social distance of 2.34 and only two matching features from the set $C$ of characteristics, each being assigned the importance of $50 \%$ and $80 \%$. Then, the social distance would be calculated by the weighted sum of those, that is, $2.34 \times(0.5 \times 0.8)=0.936$.

In this way, a homophily computation function is implemented where a given original social distance between two members is reduced by a degree that is equal to the combined effects of each matching homophily feature. Unmatched features between two members have no value; therefore, are excluded from the calculations

\section{PROTOTYPE AND VALIDATION}

The proposed recommender model was implemented on a commercial spreadsheet program. In this study, Microsoft Excel software is selected for the implementation of the proposed model because of its ease of use and powerful programming features.

Using the Visual Basic language of Microsoft Excel, various procedures were coded to form a complete temporal- and interaction-aware application. These developments involved a substantial effort in coding the several components and providing a user interface.

Since no work in the literature can be used for comparison purposes of the temporal recommender model and social quantifier, a hypothetical case study data is used to demonstrate the capabilities of the developed framework, which is explained in the upcoming section.

\subsection{Prototype capabilities: A case study}

In order to better understand the strengths and weaknesses of the proposed methodology, experimenting with real life's data is highly desirable. However, due to the limitations of time and effort required to acquire, prepare, and utilize such data, alternative solutions were sought. The most appropriate option 
was to search for an online and open community of practice and investigate their functionalities in terms of members' interconnectivity, interactions, and recommendations to choose the one that could render our model suitable data for practical demonstration. The Eyeknowledge.net online community met those requirements.

The Eyeknowledge.net is a virtual community where players from the eye care industry come together to share their various knowledge in the topic though online discussions. Each of the different types of member shares a public profile with sociodemographic, areas of interest and specialties; thus, being very suitable to derive members' data. The homophily data entered into the model are given in (Table 1). Moreover, members engage in feverous and continuous online discussions on several related themes that even include a voting system where they elicit their preference to a member's post. These features helped deriving data for the interactions over time, the social contexts, and the recommendation statements from one member to another and in which context they were inserted. The data in Table 2 represents the derived dimensions in which conversation among members occur thus was used as social contexts.

As per the proposed model's specifications, mock-up data of a complete social network based on the above was inputted into the system for experimentation. A small random social network of 35 members and 74 statements of interaction among them were used for demonstration purposes. In addition, 5,000 complete recommendation statements were also randomly generated, imported and readily made available to the model. The much larger number of recommendations is to account for the several social contexts and a 5-year recommendation period.

Table 1. List of members' profile data

\begin{tabular}{|c|c|c|}
\hline No. & Attribute & Value \\
\hline 1 & Gender & Male, Female. \\
\hline 2 & Age & $\begin{array}{l}0 \text { to } 20 \text { (Children/Youth), } 20 \text { to } 34 \text { (Younger } \\
\text { Adults), } 35 \text { to } 49 \text { (Adults), } 50 \text { to } 65 \text { (Older Adults), } \\
\text { Over } 65 \text { (Seniors). }\end{array}$ \\
\hline 3 & Education & $\begin{array}{l}\text { Secondary or less, Technical/Trade training, Post } \\
\text { secondary (college, university), Post graduate } \\
\text { (Masters and above). }\end{array}$ \\
\hline 4 & Role & $\begin{array}{l}\text { Administrative Staff, Industry, MD, OD, Optician, } \\
\text { Researcher, Student/Resident, Technical Staff, } \\
\text { Patient. }\end{array}$ \\
\hline 5 & Interest & Business, Clinical, Medical. \\
\hline 6 & Specialty & $\begin{array}{l}\text { Cataract, Contact Lenses, Cornea, Cornea and } \\
\text { External Disease, Equipment, Glaucoma, Industry, } \\
\text { Neuro-ophthalmology, Ophthalmic Pathology, } \\
\text { Ophthalmic plastic surgery, Pediatric } \\
\text { Ophthalmology, Refractive Surgery, Retina, } \\
\text { Retinal physiology and pathology, Vitreoretinal } \\
\text { Diseases, Others. }\end{array}$ \\
\hline
\end{tabular}

Table 2. List of social contexts

\begin{tabular}{|c|l|l|}
\hline No. & Attribute & Description \\
\hline 1 & Lifestyle & $\begin{array}{l}\text { Any matters related to how to improve personal } \\
\text { health and well-being }\end{array}$ \\
\hline
\end{tabular}

\begin{tabular}{|c|l|l|}
\hline 2 & Drugs & $\begin{array}{l}\text { encompass all topics related to use of } \\
\text { prescription drugs, their effects and related } \\
\text { (mis)conducts }\end{array}$ \\
\hline 3 & Prognosis & $\begin{array}{l}\text { any forecast about the course or outcome of an } \\
\text { illness }\end{array}$ \\
\hline 4 & Diagnosis & $\begin{array}{l}\text { any opinions derived from the process of } \\
\text { identifying or determining the nature and cause } \\
\text { of a disease or injury through evaluation of } \\
\text { patient history, examination, and review of } \\
\text { laboratory data }\end{array}$ \\
\hline 5 & Treatment & $\begin{array}{l}\text { expressed opinions on necessary care provided to } \\
\text { improve a medical condition, procedures or } \\
\text { applications that are intended to relieve illness or } \\
\text { injury }\end{array}$ \\
\hline 6 & Business & $\begin{array}{l}\text { outlooks on services available to commercial } \\
\text { clients who offer assistance with marketing, } \\
\text { brand awareness, as well as, providing guidance } \\
\text { relating to techniques for treating various ocular } \\
\text { disorders }\end{array}$ \\
\hline
\end{tabular}

Once the network is defined, the model is ready for verification and experimentation, as described in the following subsection.

\subsection{Recommender Search}

Having defined the present conditions of a social network with the recommender and interaction models, the proposed SCRM uses a Path-based optimization algorithm [10] to determine the optimum priority list of members and their social relations conditions. More specifically, the primary goal of the recommender search technique is to guide a source member to a destination member through only the most recommended and closest members, not any members. Having such capability available for community members is very important because it can help them to avoid social pitfalls, such as Structural Holes [3], or to access their network resources through different types of ties, such weak ties [8].

As such, the procedure searches for the path with lowest cost (i.e., closest members) between a community member and every other member with respect to user defined constraints. The social closeness assessment model is the basis for the cost structure that had to be implemented, so that the path-related algorithm can function accordingly. As seen, the Closeness indicator represents the total "social distance" between a pair of members taking into consideration their interactions and recommendations in several social contexts over a certain period of time.

Implementing a graph search algorithm in the framework involves four main steps: (1) eliciting community members, their interactions and recommendations in a given period of time and social contexts; (2) setting the source and destination members; (3) deciding on the evaluation criteria, higher or lower Closeness; and, (4) applying the relaxation principle to generate short paths to display the list of members ones should follow to optimally reach the destination member. Because the cost structure is a function of the social closeness index with lower values indicating closer (more recommended) members, the search procedure was adapted to find the path with the highest cost (less recommended), thus providing greater capability.

After defining the cost structure, the constraints considered in the algorithm are (Figure 5): 


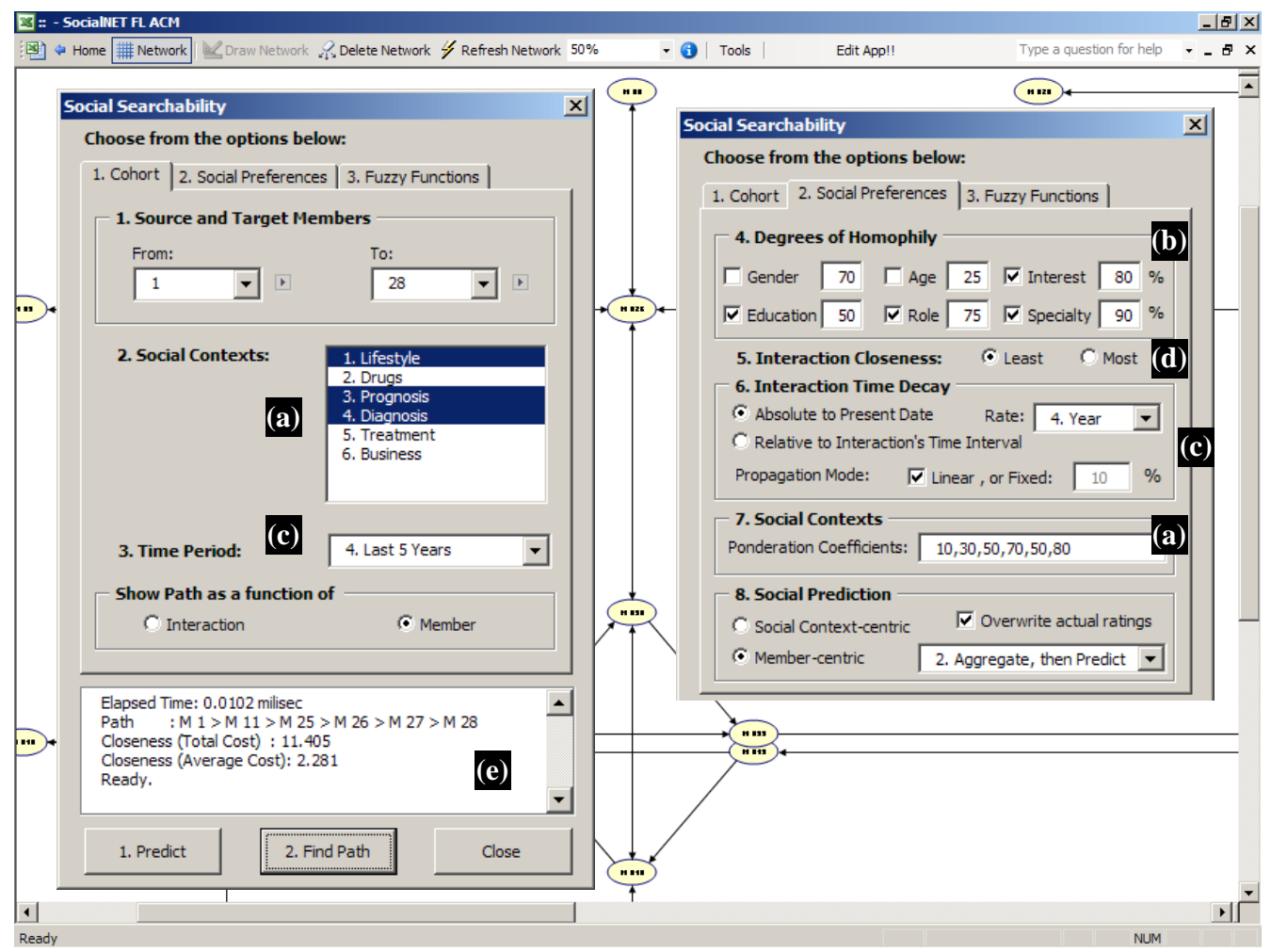

Figure 5. User-defined search strategies and network interface

a. Choose one or more Social Contexts, assigning corresponding degrees of importance - This will filter out members, interactions and recommendations that are not of interest;

b. Choose one or more homophily parameters, assigning corresponding weights - This will affect the calculation of the final Social Closeness value for each pair of member;

c. Choose time horizon and decay frequency - This concerns to the importance that old recommendations should have as compared to newer ones, ultimately affecting the combined recommender value corresponding for the whole period

d. Specify whether to use lower or higher closeness - This will affect the selection of preferred intermediate members to reach the desired destination member.

To evaluate a possible solution that gives the list of members (Figure 5.e), the recommender search algorithm identifies, analyses and builds the cost structure by using the desired constraints for a particular population. Once the target population (cohort) of that community has been created, the social distance is calculated for each interaction for all of their members. Then, beginning from the source member, paths from one member to another whose total cost is the least (or most) among all such paths is calculated until the target member is reached.

\section{DISCUSSION}

The developed framework has been demonstrated to work successfully on the example application presented in this article. Various other problems with different combinations of personal networks with different properties and different conditions were experimented with, and the system performed well.

There are a number of possible extensions to the model currently being pursued and caveats being addressed, including:

- Decay Functions: The proposed model assumed that recommendations proportionally weaken to the number of periods in the time horizon. This is the linear decay model; however, there are many other types of decay functions such as exponential and polynomial where the proportionality of the decay varies with time as well. Experimenting with different types of decay functions to evaluate their effects in the model's output is needed;

- Time History: It can be defined in any time unit, such as years, months, weeks, days, etc... which is a very powerful added feature because the more periods $h$ in the time horizon, the less sensitive the decay will be in respect to time.

- Homophily Computation: due to the lack of standardization, other formulation schemes could be sought. As described, the present model employs a socalled "All-or-Nothing" concept where the social distances between a pair of members is always shortened by a certain amount; however, it could have employed a so-called " $80-20$ " concept where matched features would shorten social distances by the larger number amount while unmatched features would lengthen them by the remaining. This could lead for more precise estimation of Closeness;

- Social Distance Calculation: presently, the model simply averages out each of a pair of member's Social 
Status; it could be the case, however, to develop and test other formulations that could potentially lead to different results. For example, a strategy so-called "lowest-wins" could be devised in which the Social distance between two connected members would be the lowest social status of the two; conversely, the "highestwins" strategy would consider the greater of the two;

- Search Algorithms: The choice to use Dijkstra's was mainly because it is a well-known, broadly accepted and flexible algorithm to implement despite its limitations (e.g., the algorithm will fail for negative Closeness). Because of that, more experimentation with other classes of algorithms is needed, which could not only improve performance (by cutting down on the size of the sub-network that must be explored) but also lead to different results;

- Collaborative Filtering: Cold-Start and data sparseness are well-known phenomena in the research literature that could hinder the effectiveness of Recommender Systems' prediction. The implications of these in the model were not considered;

For this demonstration, the size of the network was kept small to be manageable. However, more tests with real size online communities with thousands of people are welcome to extend the methodology. Other enhancements that could be made include migrating the framework to a more advanced web-based interface with improved visualization capabilities.

\section{CONCLUSION}

In this article, a recommender model was developed incorporating time-dependent and social-aware recommendations, an interaction-based social network quantifier to identify the proximity of their members, and a modified search algorithm to optimally reach members. All framework components proved to work efficiently in support of network exploration, discovery and reach decisions.

The recommender model stores rated opinions of one member to another over time and in different social contexts. These help in predicting unknown opinions more truthfully, as more factors are considered. The social network and their quantifiers, on the other hand, effectively translates the opinions of taste into the networks' closeness relationships that account for the interaction flow, improved guidance for social status and social distance computations, and user preference of interacting with similar or dissimilar members. Lastly, the search algorithm proved to work successfully for practical size problems to find members close or apart from each other. To facilitate use of the model by practitioners, the model was implemented as a prototype spreadsheet system that is easy to use. The prototype allows the user to insert, delete and update any number of members, their interactions and recommendation statements, draws the network, and automates the search optimization. A case study was used to demonstrate the capabilities of the system.

\section{REFERENCES}

[1] Baltrunas, L. 2008. Proposed architecture for a Contextaware Recommender System. In Proceedings of the ACM conference on Recommender systems. October 23-25, Lausanne, Switzerland

[2] Bott, H. 1928. Observation of play activities in a nursery school. Genetic Psychology Monographs, 4:44-88.

[3] Burt S., Ronald. 1992. Structural Holes. Chicago: Univ. Chicago Press.

[4] Capuruco R. A. C. and Capretz L. F., 2008. A Unifying Framework for Building Social Computing Applications, 1st World Summit on Emerging Technologies and Information Systems for the Knowledge Society, Athens, Greece, Lecture Notes in Computer Science - Volume LNCS/LNAI 5288/2008, Springer-Verlag, pp. 11-21

[5] Cohen, E., Strauss, M. 2006. Maintaining Time-Decaying Stream Aggregates. Journal of Algorithms. Volume 59, 1:1936, Elsevier Press.

[6] Dijkstra, E. W., 1959. A Note on Two Problems in Connexion with Graphs". In Numerische Mathematik, 1, S. 269-271.

[7] Goldberg, D. Nichols, D., Oki, B. M., and Terry, D., 1992. Using collaborative filtering to weave an information tapestry. Communications of the ACM 35(12):61-70

[8] Granovetter, Mark. 1983. The Strength of Weak Ties: A Network Theory Revisited. Journal of Sociological Theory, $1: 201-233$

[9] Harary F.,1969. Graph Theory, Addison-Wesley Publishing Company, page: 9-10.

[10] Lazarsfeld P., MertonR.K., 1954. Friendship as Social Process: A Substantive and Methodological Analysis, In Freedom and Control in Modern Society, 18-66, ed. M.Berger and C.Page, New York, Octagon Books.

[11] Loomis, C. P., 1946. Political and Occupational Cleavages in a Hanoverian Village. Sociometry. 9, 316-333.

[12] Lugano, G. Kyppö J., Saariluoma P., 2006. Designing people's interconnections in mobile social networks, International Conference on Multidisciplinary. Information Sciences \& Technologies (INSCIT), Merida (Spain).

[13] Massa, P. and Avesani, P., 2007. Trust-aware Recommender Systems. In Proceedings of the ACM conference on Recommender systems, October 19-20, Minneapolis, Minnesota, USA.

[14] Moreno, J.L., 1951. "Sociometry: Experimental Method and the Science of Society - An Approach to a New Political Orientation" Beacon House, Beacon, New York.

[15] Resnick, P., Varian, H., 1997. Recommender Systems, Introduction to Special Section of Communications of the ACM, vol. 40(3):56-58.

[16] Richardson, H. M., 1940. Community of Values as a Factor in Friendship of College and Adult Women. Journal of Social Psychology, 11:303-312.

[17] Wasserman, S., Faust, K. 1994. Social Network Analysis: Methods and Applications. New York, Cambridge University Press. 